\title{
In vivo Bio-Distribution and Toxicity Evaluation of Polymeric and Lipid-Based Nanoparticles: A Potential Approach for Chronic Diseases Treatment
}

This article was published in the following Dove Press journal: International Journal of Nanomedicine

\author{
João Fonseca-Gomes (iD) 1,2,* \\ Joana A Loureiro $\mathbb{D}^{3, *}$ \\ Sara R Tanqueiro (D) ${ }^{1,2}$ \\ Francisco M Mouro (D) ${ }^{1,2}$ \\ Pedro Ruivo 2 \\ Tânia Carvalho (iD ${ }^{2}$ \\ Ana M Sebastião (D) ${ }^{1,2}$ \\ Maria José Diógenes (iD) ${ }^{1,2 *}$ \\ Maria Carmo Pereiral-3* \\ 'Instituto de Farmacologia e \\ Neurociências, Faculdade de Medicina, \\ Universidade de Lisboa, Lisboa 1649-028, \\ Portugal; ${ }^{2}$ Instituto de Medicina \\ Molecular João Lobo Antunes, Faculdade \\ de Medicina, Universidade de Lisboa, \\ Lisboa 1649-028, Portugal; ${ }^{3}$ LEPABE - \\ Laboratory for Process Engineering, \\ Environment, Biotechnology \& Energy, \\ Faculty of Engineering, University of \\ Porto, Porto 4200-465, Portugal \\ *These authors contributed equally to \\ this work
}

Introduction: Nanoparticles (NPs), as drug delivery systems, appear to be a promising tool for prolonged therapeutic strategies as they allow a controlled drug release over time. However, most of the studies found in the literature simply contemplate the use of a single or low number of dosages with low NPs concentrations. In the context of chronic diseases, like Alzheimer's disease, cancer or human immunodeficiency virus (HIV), where the therapeutic scheme is also chronic, studies with numerous repeated dosages are often neglected. Methods: We screened different NPs, polymeric and lipid-based, in a repeated-dose toxicity study, to evaluate the safety and tissue distribution of promising nanocarriers to be used in the treatment of long-lasting diseases.

Results: After administrating 24 high concentrated doses of the selected NPs intraperitoneally (i.p.) (3 times a week for 2 months), animals have presented NPs accumulation in different tissues. However, neither toxicity, bodyweight changes nor clinical signs of disease were observed.

Discussion: This work demonstrates no general adverse effects upon the studied NPs repeated-dose exposure, indicating the most promising NPs to be used in the different therapeutic circumstances, which may be useful in chronic diseases treatment.

Keywords: long-lasting treatment, drug delivery systems, nanocarriers, liposomes, solid lipid nanoparticles, PLGA nanoparticles

\section{Introduction}

The field of nanotechnology is actively involved in the new research endeavors of this century. Academia and industry are dedicated pushing forward in understanding the potential effects of nanomaterials on biological systems. Nanomaterials have unique properties such as small size, large surface area, chemical composition and solubility which allow their widespread applications ranging from sustainable technology to healthcare and medicine. ${ }^{1-4}$ Importantly, nanoparticles (NPs) with small sizes are of high interest for drug delivery systems (DDS) to reach biological molecules/tissues/ organs, which are normally inaccessible for many synthetic pharmaceutics. Many drugs that have demonstrated efficacy in vitro and ex vivo lose beneficial effects when tested in vivo. This might be due to the difficulties related to drug delivery to the pharmacological target. To increase the bioavailability and selectivity of the compounds, NPs are considered a future strategy. ${ }^{5-8}$ Moreover, NPs are DDS that protect drugs from degradation and transport them without modifying their intrinsic properties. ${ }^{9}$ Additionally, NPs have remarkable properties, such as biodegradability,
Correspondence: Maria José Diógenes Institute of Molecular Medicine, Faculty of Medicine, University of Lisbon, Avenid Professor Egas Moniz, Lisbon 1649-028, Portugal

Email diogenes@medicina.ulisboa.pt

Maria Carmo Pereira

Faculty of Engineering, University of

Porto, R. Dr. Roberto Frias, s/n, Porto

4200-465, Portugal

Email mcsp@fe.up.pt
International Journal of Nanomedicine 2020:15 8609-862I

8609

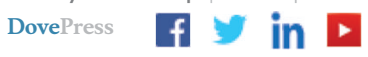

(c) (i) (5) 2020 Fonseca-Gomes et al. This work is published and licensed by Dove Medical Press Limited. The full terms of this license are available at https://www.dovepress.com/ c. the work you hereby accept the Terms. Non-commercial uses of the work are permitted without any further permission from Dove Medical Press 
biocompatibility, non-immunogenicity, metabolic stability and high selectivity to interact with specific receptors. ${ }^{10,11}$ Some of them are metal-based NPs, semiconductor NPs, polymeric NPs or lipid-based NPs. ${ }^{12-15}$ In this work, we assessed long-term distribution and toxicity of the three of the most promising ones: liposomes, poly(lactic-coglycolic acid) (PLGA) NPs and Solid Lipid NPs (SLN). Liposomes are small spherical-shaped vesicles that can be produced from natural nontoxic phospholipids and cholesterol. ${ }^{16}$ These amphiphilic assemblies are composed of phospholipid bilayers that are concentrically oriented around an aqueous compartment. These nanocarriers encapsulate hydrophilic or hydrophobic molecules. ${ }^{17,18}$ Liposomes vary in sizes, from small unilamellar vesicles (diameter $<100 \mathrm{~nm}$ ) to multilamellar vesicles with a mean diameter between 100 and $1000 \mathrm{~nm}$. They are promising DDS, because of their small size, fluidity, permeability, hydrophobic/hydrophilic character and composition (concentration of the phospholipids). Liposomes have also the ability to fuse with cell membranes, granting the drug release into the cells. PLGA is a well-established biodegradable polymer, produced by ring-opening co-polymerization of lactide and glycolide. PLGA NPs have been a center of attention in the nanomaterials field due to its appealing properties including biocompatibility and biodegradability, Food Administration and Drug (FDA) approval, quick adaption towards hydrophobic or hydrophilic small molecules, and high selectivity to target specific cells or organs. ${ }^{19}$ These NPs have various biomedical applications, such as in cancer, diagnosis, imaging, vaccination, transportation of anti-inflammation drugs and transportation of drugs for several diseases treatment. ${ }^{20-22}$ SLNs are nanocarriers comprised of a solid hydrophobic lipid core that permits the encapsulation of hydrophobic drugs. These nanocarriers present a size between 40 and $1000 \mathrm{~nm}$, allowing their transportation in body fluids. ${ }^{23}$ They are composed of solid fat (approximately $0.1-30(\% \mathrm{w} / \mathrm{w}))$ which is dispersed in the aqueous phase. ${ }^{24}$ Surfactants are used to enhance the stability of SLNs. The lipids used for SLNs preparation can be fatty acids, monoglycerides, diglycerides and triglycerides, etc. The selection of these components (including both lipids and surfactants) can influence the particle size, drug loading, release profile and long-term stability. $^{24}$ Since SLNs are composed of lipids that are biocompatible and biodegradable, they exhibit good release profile and have other advantages like targeted drug delivery with excellent physical stability. Hence, they have proved suitable for brain targeting and cancer. ${ }^{25-27}$
The remarkable physicochemical properties and the potential biological applications of these nanocarriers already addressed many challenges in the field of medicine. Despite the advancement in the field of nanotechnology, solid studies that evaluate the long-term use of these NPs through intraperitoneal (i. p.) administration route are missing in the literature.

The present study is focused on the evaluation of the NPs toxicity and accumulation in different tissues, such as brain, lung, kidneys, heart, liver, spleen, bone marrow and thymus, after 24 doses administration i. p., 3 times a week, using high NPs concentrations. The data herein reported while screening different NPs demonstrate their possible application in the context of several chronic diseases, like Alzheimer's disease, cancer, cystic fibrosis, epilepsy, HIV, among others that require prolonged drug administration.

\section{Materials and Methods Nanoparticles Formulation Solid Lipid Nanoparticles}

To produce SLNs, cetyl palmitate (2 g) (Gattefossé, Lyon, France) was used as the solid lipid and Pluronic ${ }^{\circledR}$ F-127 (from Sigma-Aldrich, St Louis, MO, USA) 10\% (w/v) $(17.4 \mathrm{~mL})$ as surfactant, that acts as a coating of the solid hydrophobic lipid core. ${ }^{28,29}$ The lipid and aqueous phase were heated at $70^{\circ} \mathrm{C}$ separately until the lipid was melted. Then, the lipid phase was dispersed in the aqueous phase. To control the diameter of the particles, the solution was exposed at a high-speed stirring in an Ultra-Turrax T25 (Janke and Kunkel IKA-Labortechnik, Staufen, Germany), for 2 minutes at 13,500 rpm followed by sonication using a Vibra-Cell ${ }^{\mathrm{TM}}$ CV18 (Sonics and Materials, Newtown, CT, USA) with an amplitude of $70 \%$ for 30 minutes and cooled down to RT.

\section{Liposomes}

For the liposomes production, all the lipids were purchased from Avanti Polar Lipids (Alabaster, AL, USA). The liposomes were produced using the thin lipid film hydration method. ${ }^{30}$ Briefly, the lipids DSPC (1,2-distearoyl-sn-glycero-3-phosphocholine), Chol (cholesterol ovine wool), DSPE-PEG 2000 (1,2-distearoyl-sn-glycero-3-phosphoethanolamine- $N$-[amino(polyethylene glycol)-2000] ammonium salt) in a molar ratio of 52:45:3 dissolved in chloroform are mixed, and then, the solvent is removed by evaporation using a nitrogen stream. The resultant dried lipid film was dispersed in PBS buffer, $\mathrm{pH} 7.4$ (phosphate buffered saline, $10 \mathrm{mM}$ phosphate buffer, $2.7 \mathrm{mM}$ 
potassium chloride and $137 \mathrm{mM}$ sodium chloride, SigmaAldrich, St. Louis, MO, USA). The liposomes suspension was then sonicated using a Vibra-CellTM CV18 (Sonics and Materials, Newtown, CT, USA) with an amplitude of $40 \%$ for 20 minutes (one-minute on/one-minute off).

\section{Poly(Lactic-Co-Glycolic Acid) Nanoparticles}

PLGA NPs were synthetized by the single emulsion-solvent evaporation method, using poly(vinyl alcohol) (PVA) (Mowiol $^{\circledR}$ 4-88, Sigma-Aldrich, St Louis, MO, USA) as a stabilizer. ${ }^{31}$ PLGA (PLGA Risomer ${ }^{\circledR}$ RG503H 50:50, Sigma-Aldrich, St Louis, MO, USA) was dissolved in ethyl acetate solution (Sigma-Aldrich, St Louis, MO, USA) and placed for 5 minutes in an ultrasonic bath at RT at $45 \mathrm{kHz}$ (Ultrasonic cleaner, $\mathrm{VWR}^{\mathrm{TM}}$, Kuala Lumpur, Malaysia). A $1 \%(w / v)$ of PVA (Sigma-Aldrich, St Louis, MO, USA) was added drop by drop to the previously prepared organic solution. After vortexion (Genius 3, Ika ${ }^{\circledR}$ vortex, Germany), the oil in water (o-in-w) solution was sonicated three times for one minute (10 seconds on/10 seconds off) on ice. Further, the solution was maintained in continuous agitation (600 rpm) at RT until complete organic solvent evaporation (4 h). Finally, the NPs were lyophilized during $24 \mathrm{~h}$ using a benchtop vacuum concentrator (VirTis freeze-dryer (SP Scientific, NY, USA)) at $-90.2^{\circ} \mathrm{C}$ (condenser temperature) and $8 \mathrm{~mm}$ Torr of vacuum pressure. The lyophilized PLGA NPs were stored at $4^{\circ} \mathrm{C}$. Before using, the PLGA NPs were dissolved in ultrapure water at a final concentration of $10 \mathrm{mg} / \mathrm{mL}$ and ultrasonicated for 5 minutes (RT at $45 \mathrm{kHz}$ ), for complete dispersion of the NPs in the suspension.

\section{Nanoparticles Physicochemical Characterization}

The physicochemical features of each type of NPs, as hydrodynamic diameter, size distribution and zeta potential, were analyzed by dynamic light scattering (DLS) using a Zetasizer Nano ZS (Malvern Instruments Ltd.). The zeta potential was obtained by using the dielectric constant of water and the zeta potential values were estimated by Smoluchowski approximation from the electrophoretic mobility. ${ }^{32}$ Before each measurement, samples were diluted 200x in ultra-pure water (SLN and PLGA NPs) or PBS buffer (liposomes) before each measurement.

Morphological analysis of the NPs was attained by Transmission Electron Microscopy (TEM) using a JEM 1400 electron microscope (Jeol, Tokyo, Japan) at $80 \mathrm{kV}$. Five microliters of each NPs sample was deposited on copper grids (Formvar/Carbon-400 mesh Copper, Agar
Scientific, UK) for 5 minutes. Samples were negatively stained with a $2 \%(\mathrm{v} / \mathrm{v})$ uranyl acetate solution for 45 seconds.

\section{Animals}

Adult (3 months old, m. o.) male and female C57BL/6 mice were obtained from Charles River, Barcelona, Spain. All procedures regarding animal manipulation followed the European Community Guidelines (Directive, 2010/63/EU) and the Portuguese law (DL 113/2013) for Animal Care for Research Purposes and were approved by the "Instituto de Medicina Molecular - João Lobo Antunes" Internal Committee (AWB_2016_17_SX_Adenosina_Addendum). Animals were housed in a room with regulated temperature $\left(22-24{ }^{\circ} \mathrm{C}\right)$ and humidity (45-65\%) with ad libitum access to food and water and with a 14/10 h light/dark cycle. A total of 31 animals were randomly allocated to the different treatment groups: control (not injected), 4 males and 3 females; Lipo, 4 males and 4 females; PLGA, 4 males and 4 females; SLN, 4 males and 4 females. Animals were tagged and randomly distributed in groups of 4 animals per cage ( 3 animals in the case of control females).

\section{Nanoparticles Administration}

NPs were administered in $3 \mathrm{~m}$. o. animals by i. p., three times per week at a dose of $100 \mathrm{mg} / \mathrm{kg}$, for 56 days (in a total of 24 injections per animal). NPs administration injections were performed during the light phase, around the same time each day.

\section{Clinical Evaluation}

Mice were screened three times a week for clinical signs of toxicity, morbidity, and mortality. Animal bodyweight (BW) was also assessed in three different time-points: before starting the administration protocol, on day 30 after first administration (mid-point) and immediately before euthanasia (end-point). Since the initial values for bodyweight were not exactly similar for all conditions, group comparisons of absolute weight were considered inadequate (Supplementary Figure 1). In this way, animal $\mathrm{BW}$ changes $(\triangle \mathrm{BW})$ for each animal were calculated by subtracting the initial weight to that at mid-point and endpoint, with results being expressed in grams $(\mathrm{g})$.

\section{Histopathology}

Three days after the last injection, mice were euthanized with anesthetic overdose and comprehensive necropsy was performed. Macroscopic findings were recorded, and 
brain, lung, kidneys, heart, liver, spleen, bone marrow and thymus were collected for histopathology. Samples were immersion fixed in 10\% neutral buffered formalin, routinely processed for paraffin embedding, sectioned at $4 \mu \mathrm{m}$, and stained with hematoxylin and eosin. Lesions were classified according to previously published criteria and scored according to a 5-tier severity scale: 0 , absent; 1 , minimal; 2, mild; 3, moderate; 4 , marked. ${ }^{33}$ Representative microphotographs were obtained using NDP.view2 software (Hamamatsu) in slides digitally scanned in the Hamamatsu NanoZoomerSQ, at 20x magnification.

Birefringence of NPs under polarized light was used to assess their distribution and accumulation, scored according to the same 5-tier severity scale presented above. Representative microphotographs were taken in Leica DM2000 microscope coupled to a Leica MC170 HD microscope camera (Leica Microsystems, Wetzlar, Germany) at 20x and 40x original magnifications.

\section{Statistical Analysis}

Data were analyzed through a one-way analysis of variance (ANOVA) with Holm-Sidak's correction for multiple comparisons where it is needed. All comparisons were made between groups, with a statistical significance level $(\alpha)$ established at $p$-value $<0.05$. Data are expressed as means \pm standard error of mean (SEM). All statistical analyses were performed using GraphPad Prism 8 software (GraphPad Software, San Diego, CA, USA).

\section{Results}

\section{Nanoparticles Physicochemical Characterization}

Three different types of NPs, liposomes, PLGA NPs and SLNs, were prepared and analyzed in this work according to their size, polydispersity index and zeta potential.

NPs size is one of the most relevant factors that influences the NPs efficiency. ${ }^{34,35}$ This parameter determines if the NPs are able to cross different types of biological barriers, such as BBB. ${ }^{36}$ NPs size is also important to predict the bio-distribution and accumulation in different tissues. All produced NPs present a size lower than or around $200 \mathrm{~nm}$, which is the maximum size required to cross most of the biological barriers. ${ }^{37}$ Liposomes size is significantly smaller than SLN and PLGA NPs (Table 1). Liposomes present a mean diameter of $96 \mathrm{~nm}$, and the PLGA NPs and SLN are closer to $200 \mathrm{~nm}$ (183 nm and 203 nm, respectively) (Figure 1A-C). Previously, we proved that this type of liposomes is stable for 2 months. ${ }^{30}$

Polydispersity is another important parameter to evaluate if the NPs population is homogeneous. The prepared nanocarriers exhibit a small size distribution closer or lower than 0.2 , suggesting a homogeneous size distribution (Table 1) ${ }^{38}$ Liposomes are the ones that present higher polydispersity index (PdI), which may be due to the fabrication protocol. The ultrasonication method used to produce liposomes appears very promising since it is faster in comparison to extrusion (the most common method used in the liposomes fabrication). However, it is difficult to achieve homogeneity in NPs' size via sonication method. ${ }^{39}$ On the other hand, PLGA NPs present a very homogeneous population, with a polydispersity index of 0.07 .

Zeta potential is an extremely important parameter to predict the stability of colloidal dispersions. ${ }^{40}$ Zeta potential is the potential difference between the phase boundaries of solids and liquids. It is a measure of the electric charge of particles that are suspended in liquids. A high zeta potential reflects stability, i.e, the dispersion will resist to aggregation. If the potential is small, the attractive forces will overcome and the dispersion may break or flocculate. According to the results depicted in Table 1, SLN and PLGA NPs have a negative zeta potential, around $-20 \mathrm{mV}$, indicating a rather high value to prevent NPs aggregation. In the case of PLGA NPs, the zeta potential is negative, as it is expected due to the negative charge of the carboxylic groups of PLGA polymer. SLNs also present negative zeta potential, due to

Table I SLN, Liposomes and PLGA NPs Stability According to Their Size, Polydispersity Index and Zeta Potential. Results are Presented as Mean \pm SD $(n=3)$

\begin{tabular}{|c|c|c|c|c|c|c|c|c|c|}
\hline & \multicolumn{3}{|c|}{ Particle Size (nm) } & \multicolumn{3}{|l|}{ PdI } & \multicolumn{3}{|c|}{ Zeta Potential (mV) } \\
\hline & Day 0 & Day 3 & Day 7 & Day 0 & Day 3 & Day 7 & Day 0 & Day 3 & Day 7 \\
\hline Liposomes & $96 \pm 9$ & $97 \pm 8$ & $97 \pm 3$ & $0.21 \pm 0.02$ & $0.22 \pm 0.05$ & $0.22 \pm 0.02$ & $-1.2 \pm 0.8$ & $-2.0 \pm 0.8$ & $-0.9 \pm 0.6$ \\
\hline PLGA NPs & $183 \pm 9$ & $182 \pm 9$ & $180 \pm 9$ & $0.07 \pm 0.01$ & $0.06 \pm 0.02$ & $0.07 \pm 0.02$ & $-21.2 \pm 0.5$ & $-20 \pm 2$ & $-19 \pm 2$ \\
\hline SLNs & $203 \pm 7$ & $197 \pm 4$ & $199 \pm 6$ & $0.12 \pm 0.02$ & $0.12 \pm 0.01$ & $0.13 \pm 0.02$ & $-17 \pm 3$ & $-18 \pm 3$ & $-16 \pm 3$ \\
\hline
\end{tabular}

Abbreviation: Pdl, polydispersity index. 

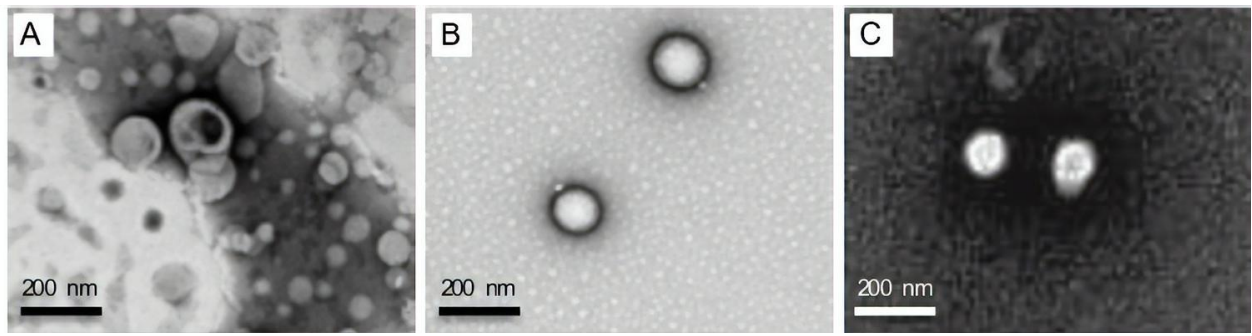

Figure I Morphological characterization of NPs by transmission electron microscopy: liposomes (A), PLGA NPs (B) and SLN NPs (C). Samples were diluted at a ratio of I:I00. Scale bar corresponds to $200 \mathrm{~nm}$.

the cetyl palmitate nature. Liposomes present a zeta potential close to $0 \mathrm{mV}$, which is expected due to the lipids nature.

Every week, fresh NPs were produced. That way, the stability of NPs was monitored for 7 days and no significant alterations in size, PdI or zeta potential was observed (Table 1). Additionally, PVA was used as an emulsion stabilizer for PLGA NPs since it has a high affinity towards PLGA. PVA forms a uniform layer on the NPs' surface, conferring stability against aggregation due to the steric repulsion between NPs. ${ }^{41}$ Also, Pluronic ${ }^{\circledR}$ F-127 works as a stabilizer for SLNs.

\section{Clinical Evaluation}

There were no unscheduled deaths, and no systemic clinical signs of disease in any of the animals. To evaluate whether animal manipulation, injection-associated stress or NPs administration could affect animal BW, this parameter was monitored for the duration of NPs administration. To decrease animal stress during the $\mathrm{BW}$ measurements, animals were weighed in three crucial moments: prior to the first NPs injection (3 m. o.), in the middle of the injection period ( $4 \mathrm{~m} . \mathrm{o}$.) and before mice euthanasia ( $5 \mathrm{~m}$. o.), as schematically presented in Figure 2.

Overall, we observe that the chronic injection of NPs did not affect the global BW of the animals (Figure 3). However, it should be noted that SLN-injected animals presented a transient loss in BW in the intermediate timepoint. This slight difference is indeed significant for females (Figure 3B; $\mathrm{n}=3-4 ;{ }^{*} \mathrm{p}<0.05$ when comparing SLN group to PLGA-treated animals; $* * p<0.01$, when comparing SLN group to CTR and liposomes-treated animals; one-way ANOVA with Holm-Sidak's post-test) and it is presented only by a tendency in male animals (Figure $3 A ; n=4$ ). Nevertheless, animals injected with SLN recovered BW in the last month of injections, demonstrating that NPs injection did not promote marked BW alterations for the complete time course of the experiment.

\section{NPs Systemic Distribution}

At the end of the injection period (3 days after the last injection), mice were necropsied and all major organs were collected and processed for histopathological analysis.

\section{A. Experimental Timeline}

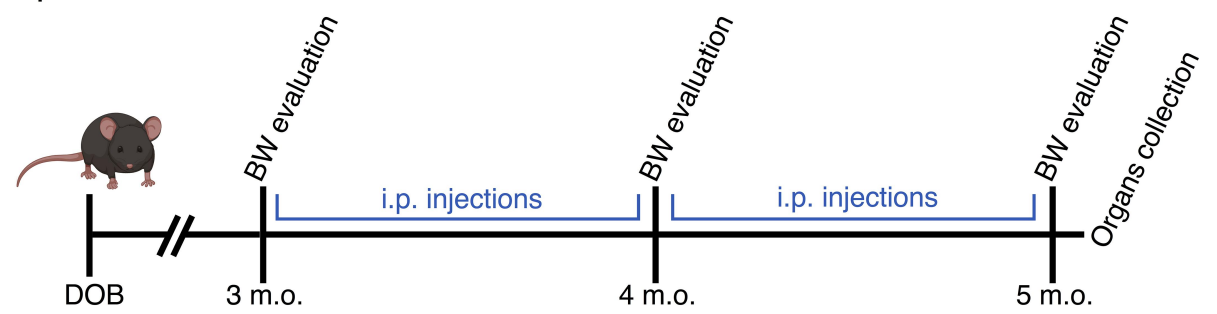

B. Organs Collected

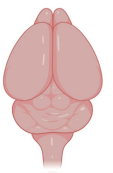

Brain

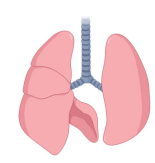

Lung

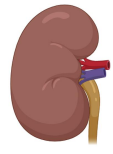

Kidney

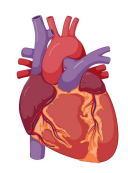

Heart

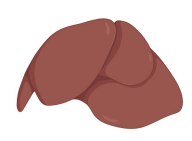

Liver

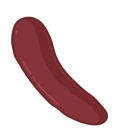

Spleen

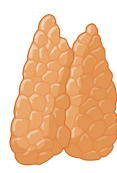

Thymus

Figure 2 (A) Experimental time-points associated with NPs injections and BW evaluation, as well as the organs collection. (B) Identification of the collected organs that were subsequently analyzed to test the presence and distribution of NPs and/or histopathological lesions. 

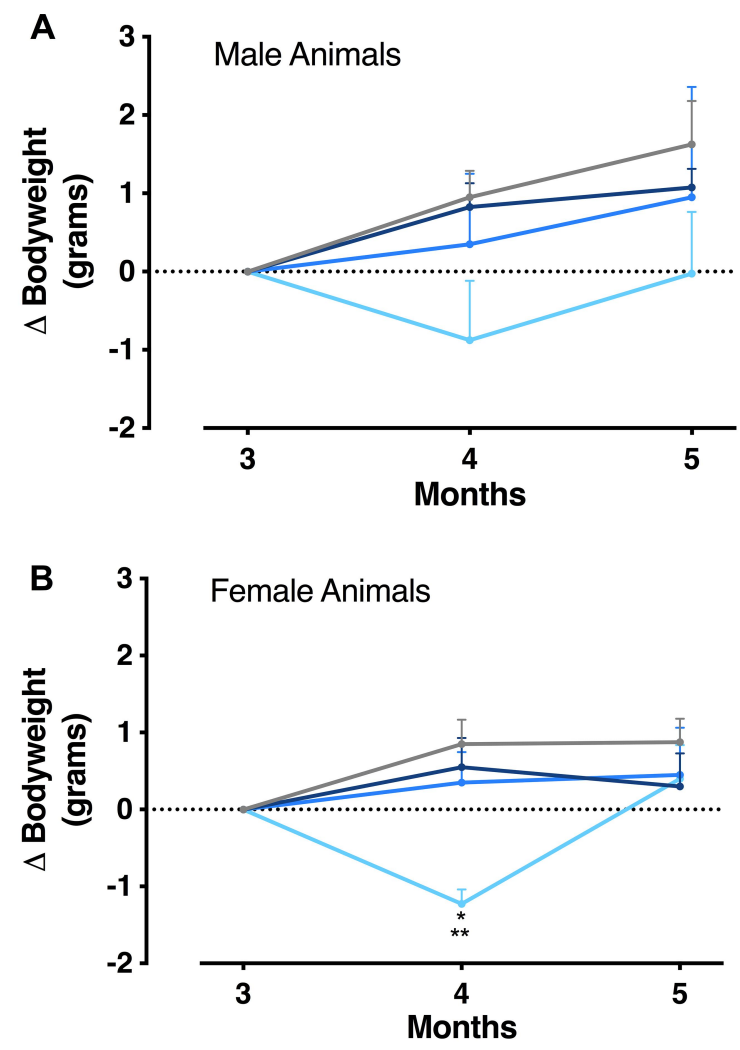

CTR

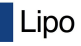

PLGA

SLN

Figure 3 (A) Bodyweight variation over experiment duration ( $x$-axis). The $y$-axis represents the weight change of male animals in relation to the first day of NPs administration. (B) Bodyweight variation over experiment duration ( $x$-axis). The $y$-axis represents the weight change of female animals in relation to the first day of NPs administration. Data are expressed as mean \pm SEM ( $n=3-4$; * $p<0.05$ when comparing SLN group to PLGA-treated animals; **p <0.0I, when comparing SLN group to CTR and liposomes-treated animals; one-way ANOVA with Holm-Sidak's post-test).

The optical birefringence of NPs allowed for scoring its tissue distribution and density. NPs were detected inside phagocytes and also extracellularly, in liver, spleen and bone marrow (Figures 4 and 5). No NPs accumulation could be detected in the brain, lung, kidney, heart, and thymus or in any organ of mice from injected animals. In NPs-injected mice, we observed that the compounds accumulated in different magnitudes, in different organs and with minor differences regarding animal sex (Figures 4 and 5). Overall, we observed that SLN-treated animals significantly showed the highest NPs accumulation, while liposomes-treated animals presented the lowest NPs accumulation and PLGA NPs-treated animals presented intermediate NPs accumulation, as described in detail below. In liver, only SLN-treated animals showed NPs accumulation, and this could be detected in both sexes (Figures 4A and 5; $\mathrm{n}=3-4 ; * * * \mathrm{p}<0.0001$; One-Way ANOVA followed by
A Liver

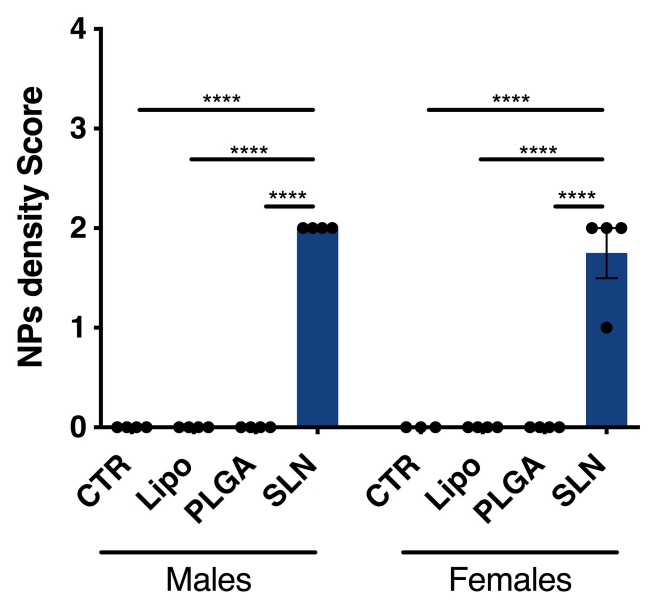

B
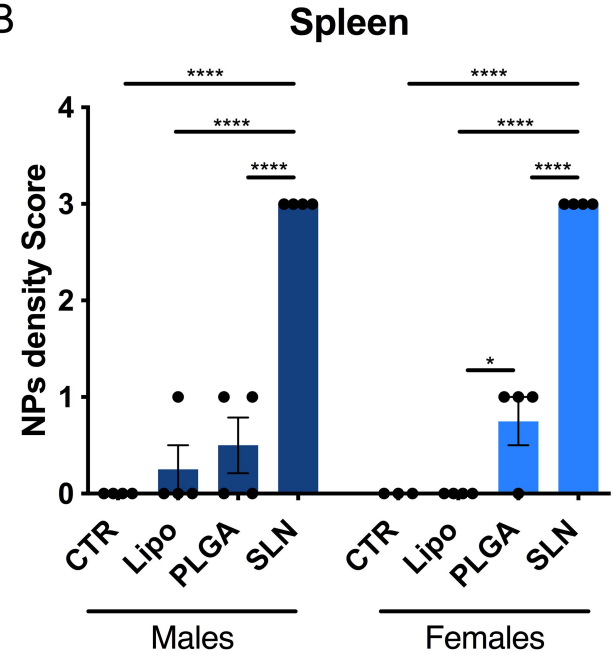

C

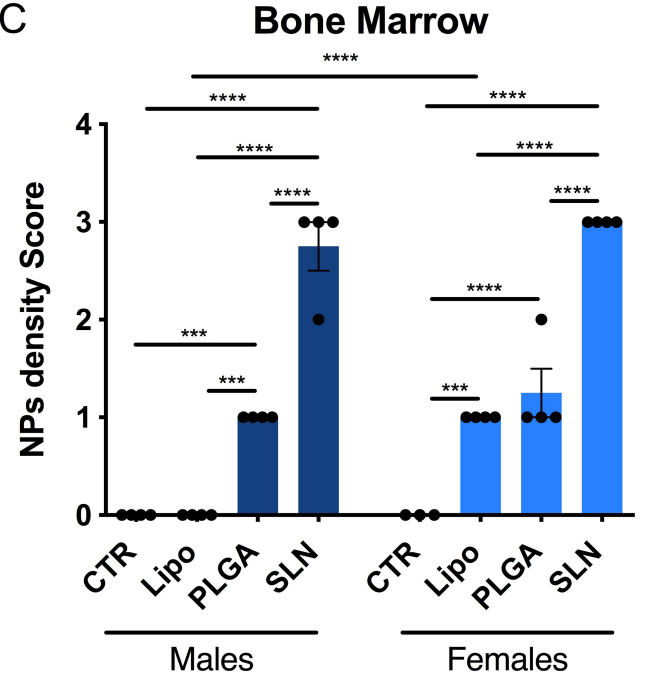

Figure 4 NPs distribution in liver, spleen and bone marrow of NPs-injected mice. Score of NPs presence and distribution in liver (A), spleen (B) and bone marrow (C) of experimental animals. Pathology score for NPs density followed a 5-tier severity scale: 0 , absent; I, minimal; 2, mild; 3, moderate; 4, marked. Data is represented as mean \pm SEM $(n=3-4$; *** $\mathrm{p}<0.001$, **** $\mathrm{p}<0.0001$; one-way ANOVA followed by Holm-Sidak's post-test). 


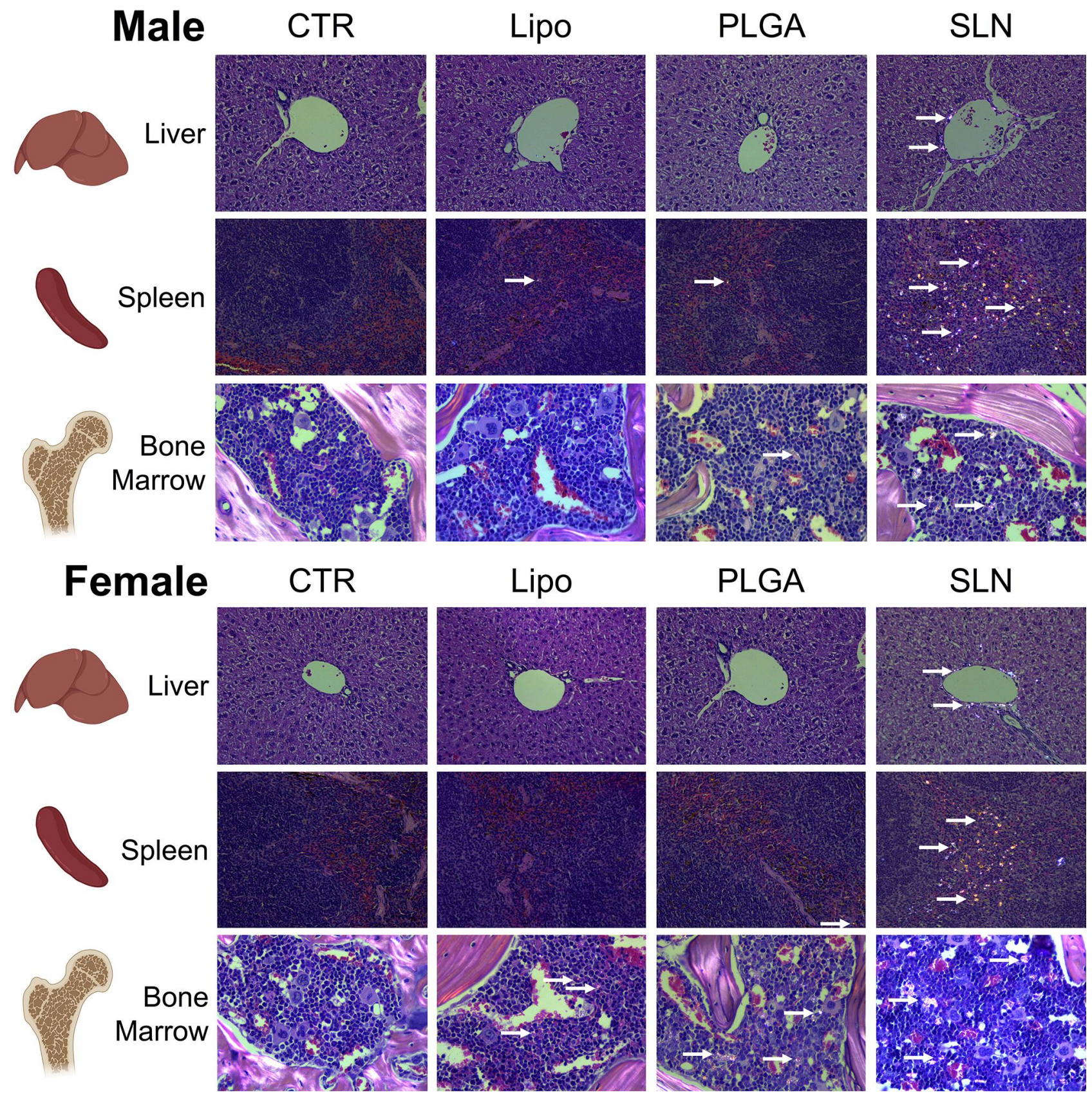

Figure 5 Histopathology images under polarized light of liver, spleen and bone marrow of CTR and NPs-injected male and female animals. NPs were not detected in organs or in control mice. Original magnification, 20x (for liver and spleen) and 40x (bone marrow). White arrows point to nanoparticles accumulated in the tissue.

Holm-Sidak's post-test). In spleen, although NPs accumulation observed in liposomes- and PLGA NPs-treated animals, there was a markedly stronger NPs accumulation in the SLN group (Figures 4B and 5; $\mathrm{n}=3-4 ;{ }^{* * * *} \mathrm{p}<0.0001$; One-Way ANOVA followed by Holm-Sidak's post-test). In this organ, an increase in NPs density in female PLGAtreated animals, as when compared to female liposomestreated animals was also detected (Figures $4 \mathrm{~B}$ and $5 ; \mathrm{n}=4$; ${ }^{*} \mathrm{p}<0.05$; One-Way ANOVA followed by Holm Sidak's post-test). Finally, in bone marrow, there was a higher NPs accumulation in the SLN group (Figures $4 \mathrm{C}$ and 5; $\mathrm{n}=3-4$; $* * * * p<0.0001$; One-Way ANOVA followed by HolmSidak's post-test). In the same tissue, we also observed a moderate NPs distribution in PLGA-treated animals (Figures 4C and 5; $\mathrm{n}=3-4 ; * * * \mathrm{p}<0.001, * * * * \mathrm{p}<0.0001$; One-Way ANOVA followed by Holm-Sidak's post-test). Interestingly, females treated with liposomes presented NPs accumulation in the bone marrow, whereas in male animals no NPs could be detected (Figures $4 \mathrm{C}$ and 5; $\mathrm{n}=4$; $* * * * \mathrm{p}<0.0001$; One-Way ANOVA followed by Holm- 
A Liver

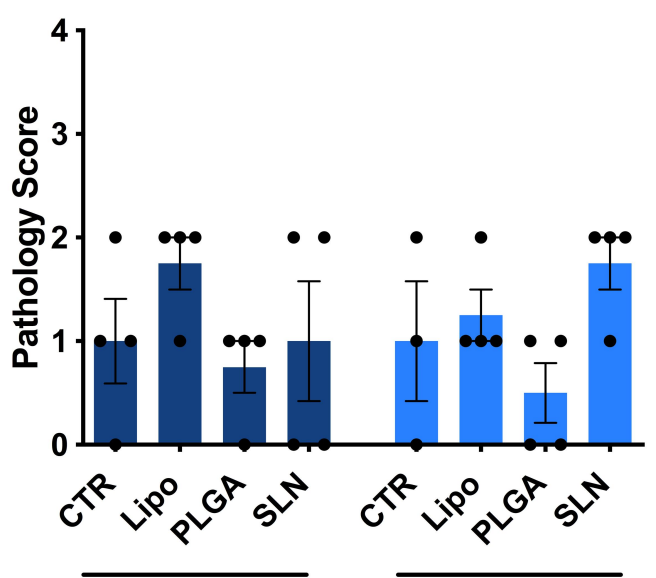

Males

Females

B

Kidney

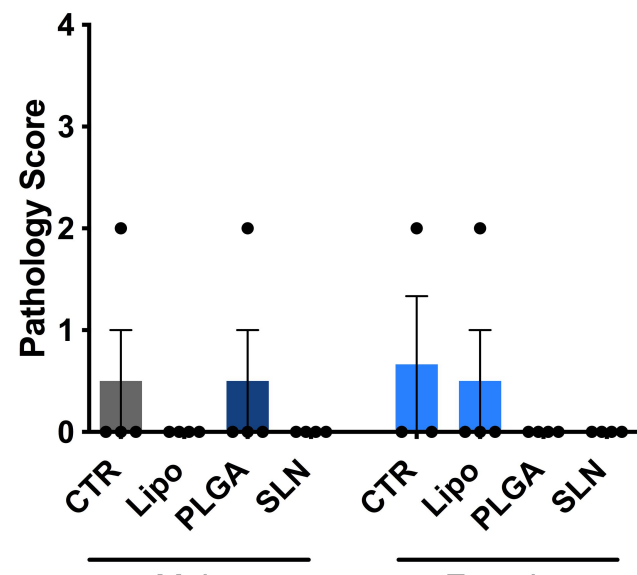

Males

Females

C

Lung

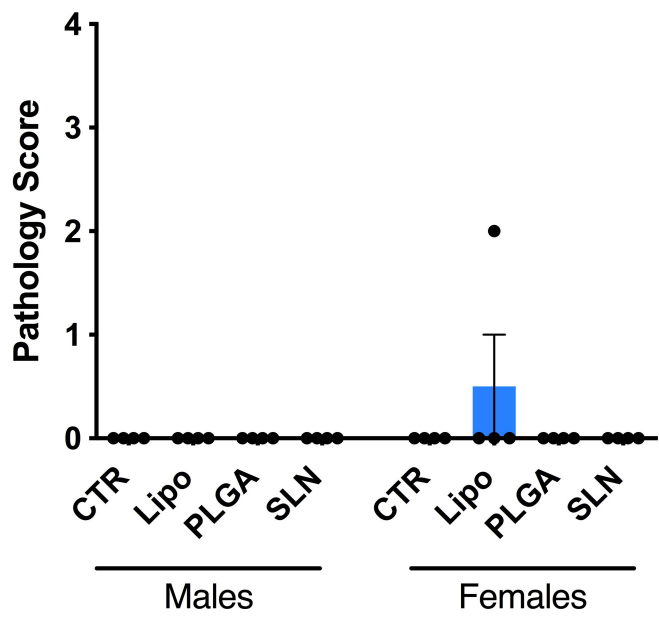

Figure 6 Identification of lesions presence and severity in liver (A), kidney (B) and lung (C) of experimental animals. No statistical differences between groups were obtained. No statistical differences between groups were obtained. Pathology score for lesions presence followed a 5-tier severity scale: 0 , absent; I, minimal; 2, mild; 3, moderate; 4, marked. Data is represented as mean \pm SEM ( $n=3-4$; non-significant; one-way ANOVA followed by Holm-Sidak's post-test). Complete description for histopathological findings is showed in Supplementary Table I. 
Sidak's post-test). This was the only statistically significant difference observed when comparing the two sexes for the same treatment and organ.

\section{Histopathology and Toxicology}

To evaluate the toxicity of NPs, histological changes were assessed in brain, heart, spleen, thymus, liver, kidney and lung (Figures 6-8). No significant changes were detected in brain, heart, spleen and thymus of both control mice or mice injected with NPs (Supplementary Table 1).

In liver and kidney, both control and NPs-injected mice showed minimal to mild lesions, corresponding to multifocal hepatocellular necrosis and mononuclear inflammatory cell infiltration of the renal stroma, seen in $77 \%$ of the animals (Figures 6A and B, 7 and 8; n=3-4; non-significant ; One-Way ANOVA followed by Holm-Sidak's post-test).
These correspond to background lesions (incidental/spontaneous findings typical in laboratory rodents), unrelated to NPs administration and with no significant difference between experimental groups ( $\mathrm{n}=3-4$; non-significant; One-Way ANOVA followed by Holm-Sidak's post-test). In the lung, focal pleuritis (mononuclear inflammatory cell infiltration, mild) was observed in only 1 out of 31 animals (Figures 6C, 7 and 8; n=3-4; non-significant; One-Way ANOVA followed by Holm-Sidak's post-test).

A complete description of the histopathological findings is showed in Supplementary Table 1.

\section{Discussion}

In the present work, we observed that SLN, when administered for 2 months, clearly accumulates in the liver, spleen and bone marrow without causing measurable

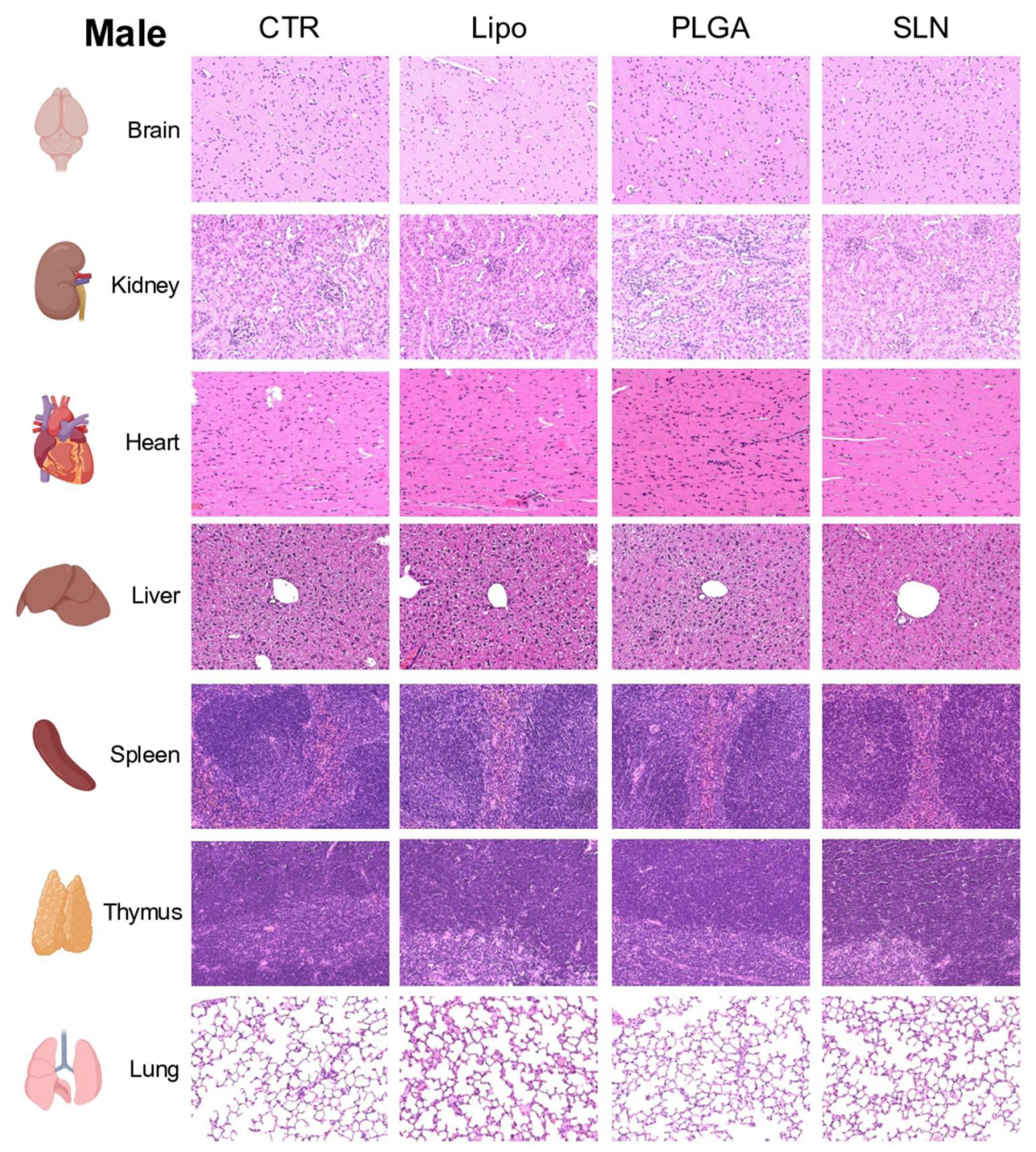

Figure 7 Representative microphotographs from male animals of H\&E-stained sections of brain, kidney, heart, liver, spleen, thymus and lung. Original magnification, 20x. 


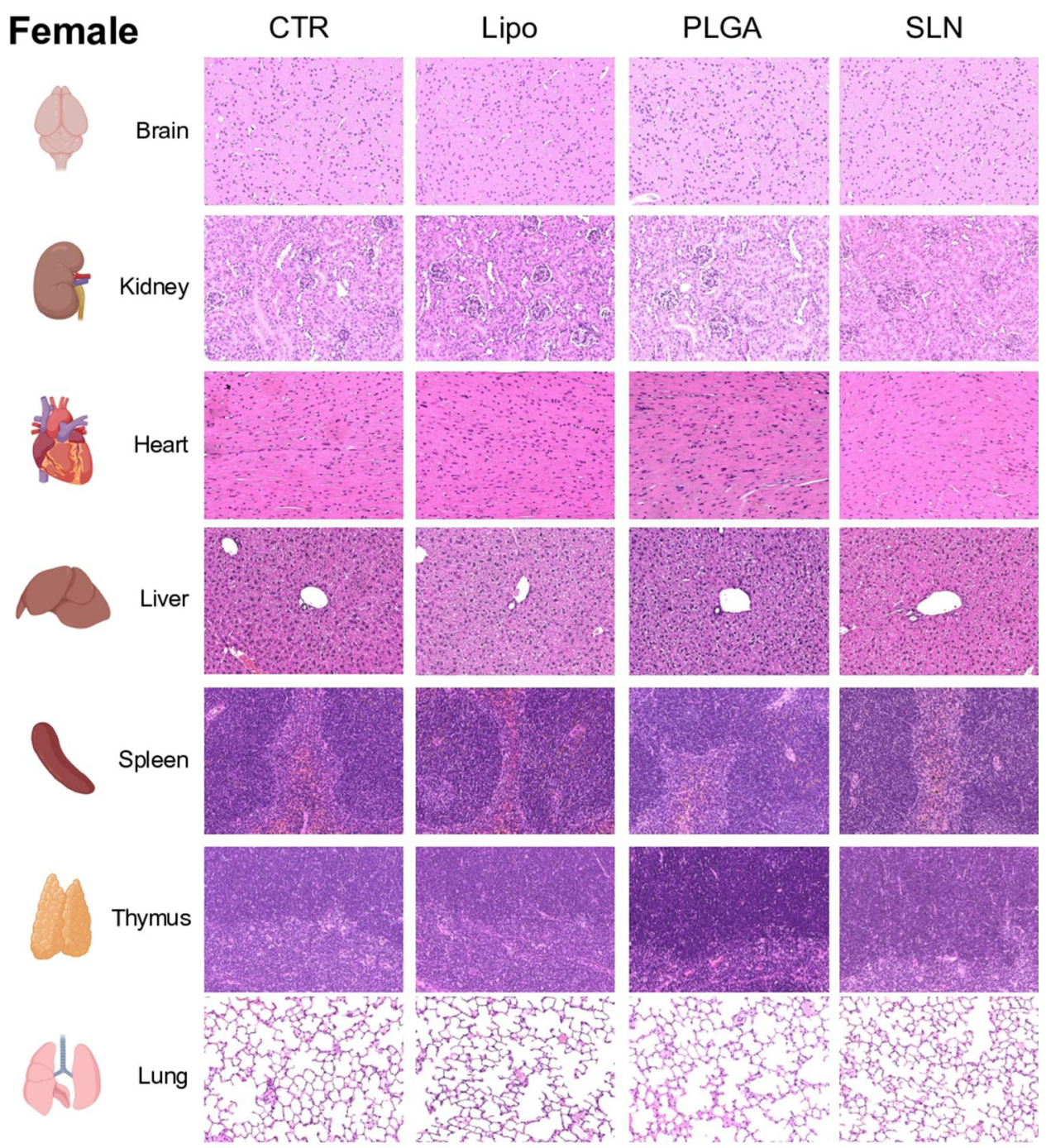

Figure 8 Representative microphotographs from female animals of H\&E-stained sections of brain, kidney, heart, liver, spleen, thymus and lung. Original magnification, 20x.

signs of toxicity. Liposome and PLGA NPs are also virtually devoid of toxicity but their accumulation is lower than SLN. Though dependent on the tissue and sex, the observed trend was that liposome nanoparticles accumulate less than PLGA NPs and always significantly less than SLN nanoparticles. However, it is important to refer that the animals treated with SLN and PLGA NPs presented some grunting noise, which may demonstrate some discomfort during NPs administration. This might be due to the SLN and PLGA NPs size since those NPs are bigger than the liposomes. Still regarding the intrinsic properties of NPs, the tendency to the reduction of BW in animals treated with SLN after 1 month of treatment may be explained by alterations in the immune system, such as its effect upon viability and cytokine production of macrophages. ${ }^{42}$ Nonetheless, the BW of SLN-treated animals is recovered and normalized at the endpoint of the study, suggesting this parameter in these animals is not impaired.

In order to evaluate the effect of NPs chronic administration, 3 days after the last injection, a comprehensive necropsy was performed to allow a deeply histopathological analysis, without the interference of possible acute signs of animal manipulation and/or i.p. injections. NPs' accumulation in tissues did not promote any toxic effect microscopically. Sex differences were almost negligible in qualitative terms, except for the accumulation of liposome NPs in the bone marrow of females but not in males. Anyway, this difference could be attributed to a multiplicity of factors that are distinct between male and female mice, including microbiota properties, genetic and hormonal events or immune cell phenotype, among others. ${ }^{43-45}$ NPs accumulation might be a useful therapeutic strategy using the NPs produced under the described protocol. Here, we should highlight the increased NPs 
accumulation for SLN-treated animals in liver, bone marrow and spleen, which may be attributed to SLN negative nature. In fact, Uner and Yener find that the hydrophobicity and the negative surface charge induce the penetration into lymphatic interstitium. ${ }^{46}$ In the future, the drug-loaded NPs that accumulate in particular tissues, releasing the drug overtime without causing toxicity for those organs, might be a strong advantage whenever these organs are the target for the delivered drug. We observed that this data is in line with previous reports, which also described liver and spleen as the dominant NPs targeted organs. ${ }^{47-52}$ Since this study did not evaluate drug-loaded NPs, one should acknowledge that the absence of toxicity needs to be demonstrated for each drug to be loaded, since it may have intrinsic toxicity. Nevertheless, our work clearly shows that there is no promotion of toxicity by the drug delivery systems tested per se. Although some evidences in the literature state that i. p. injections of several NPs could induce toxicity, detected in BW measurements. ${ }^{53}$ In this work, we did not detect any significant impact of i. p. injections by the end of the experiments.

One of the well-documented advantages of NPs is associated with a marked cellular uptake and BBB crossing. ${ }^{36,37,54-56}$ However, this should be considered, since those NPs may establish interactions with intracellular biological components, affecting their effect and possibly compromising its physiological functions overtime. ${ }^{57-60}$ Several studies were already performed to evaluate NPs toxicity; however, they are mainly answering to questions for a limited time window, ranging from an acute administration to evaluations after 4 weeks. ${ }^{53,61}$

\section{Conclusions}

In the last decades, there is a marked increase in the interest of NPs research. ${ }^{62-64}$ One of the main applications of these types of compounds, nowadays, is related to biomedicine. However, little is known about their safety in chronic studies using high dosages. The in vivo results herein reported are encouraging for long-term therapies using NPs, since these specific liposomes, PLGA NPs and SLNs, with a diameter lower than $200 \mathrm{~nm}$, did not present any measurable toxicity in the parameters and the organs evaluated. Therefore, this work highlights that the usage of NPs for longer times can be a useful therapeutic strategy. Hence, these three types of NPs studied here are of considerable interest and can be used in the treatment of chronic diseases, such as Alzheimer's and Parkinson's diseases, diabetes, cancer, cardiovascular diseases and others.

\section{Abbreviations}

ANOVA, one-way analysis of variance; BW, bodyweight; HIV, human immunodeficiency virus; m. o., months old; NPs, nanoparticles; PdI, polydispersity index; PLGA, poly (lactic-co-glycolic acid); PVA, poly(vinyl alcohol); rpm, rotations per minute; RT, room temperature; SEM, standard error of mean; SLN, solid lipid nanoparticles.

\section{Acknowledgments}

This work was financially supported by: 1) Base Funding UIDB/00511/2020 of the Laboratory for Process Engineering, Environment, Biotechnology and Energy - LEPABE - funded by national funds through the Fundação para a Ciência e a Tecnologia - Ministério da Ciência, Tecnologia e Ensino Superior (FCT-MCTES, Portugal) (PIDDAC) funded by FEDER funds through COMPETE2020 - Programa Operacional Competitividade e Internacionalização (POCI) and by national funds (PIDDAC) through FCT/MCTES; 2) Santa Casa da Misericórdia de Lisboa (MB37-2017); 3) European Union's Horizon 2020 research and innovation programme under grant agreement No 810685 (DelNAM). FCT supported J.F.G. (SFRH/PD/BD/114441/2016); S.R.T. (SFRH/PD/BD/128091/2016) and J.A.L under the Scientific Employment Stimulus - Institutional Call - [CEECINST/ 00049/2018]. All the staff from the Rodent Facility of Instituto de Medicina Molecular - João Lobo Antunes, mainly Patrícia Almeida, and Dr. Rui Fernandes from the Electron Microscopy facility of Instituto de Biologia Molecular e Celular at i3s are also acknowledged. Part of the images presented were prepared using icons provided from Biorender. Co-last authors: Maria José Diógenes and Maria Carmo Pereira. Co-first authors: João Fonseca-Gomes and Joana A Loureiro.

\section{Disclosure}

The authors report no conflicts of interest in this work.

\section{References}

1. Semete B, Booysen L, Lemmer Y, et al. In vivo evaluation of the biodistribution and safety of PLGA nanoparticles as drug delivery systems. Nanomedicine. 2010;6(5):662-671. doi:10.1016/j.nano.2010.02.002

2. Oroojalian F, Charbgoo F, Hashemi M, et al. Recent advances in nanotechnology-based drug delivery systems for the kidney. $J$ Control Release. 2020;321:442-462. doi:10.1016/j.jconrel.2020.02.027

3. Sahle FF, Kim S, Niloy KK, et al. Nanotechnology in regenerative ophthalmology. Adv Drug Deliv Rev. 2019;148:290-307. doi:10.1016/ j.addr.2019.10.006

4. Ramalho MJ, Andrade S, Loureiro JA, Do Carmo Pereira M. Nanotechnology to improve the Alzheimer's disease therapy with natural compounds. Drug Deliv Transl Res. 2019. 
5. Loureiro JA, Gomes B, Coelho MA, Do Carmo Pereira M, Rocha S. Targeting nanoparticles across the blood-brain barrier with monoclonal antibodies. Nanomedicine (Lond). 2014;9(5):709-722.

6. Nakamura T, Yamada Y, Sato Y, Khalil IA, Harashima H. Innovative nanotechnologies for enhancing nucleic acids/gene therapy: controlling intracellular trafficking to targeted biodistribution. Biomaterials. 2019;218:119329. doi:10.1016/j.biomaterials.2019.119329

7. Marques AC, Costa PJ, Velho S, Amaral MH. Functionalizing nanoparticles with cancer-targeting antibodies: a comparison of strategies. J Control Release. 2020;320:180-200. doi:10.1016/j.jconrel.2020.01.035

8. Vieira DB, Gamarra LF. Getting into the brain: liposome-based strategies for effective drug delivery across the blood-brain barrier. Int J Nanomedicine. 2016;11:5381-5414.

9. Su H, Wang Y, Liu S, et al. Emerging transporter-targeted nanoparticulate drug delivery systems. Acta Pharm Sin B. 2019;9(1):49-58.

10. Karlsson J, Vaughan HJ, Green JJ. Biodegradable polymeric nanoparticles for therapeutic cancer treatments. Annu Rev Chem Biomol Eng. 2018;9:105-127. doi:10.1146/annurev-chembioeng-060817-084055

11. Mokhtarzadeh A, Alibakhshi A, Yaghoobi H, Hashemi M, Hejazi M, Ramezani M. Recent advances on biocompatible and biodegradable nanoparticles as gene carriers. Expert Opin Biol Ther. 2016;16 (6):771-785.

12. Gajbhiye KR, Pawar A, Mahadik KR, Gajbhiye V. PEGylated nanocarriers: a promising tool for targeted delivery to the brain. Colloids Surf B Biointerfaces. 2020;110770.

13. Zheng Y, Li Z, Chen H, Gao Y. Nanoparticle-based drug delivery systems for controllable photodynamic cancer therapy. Eur J Pharm Sci. 2020;144:105213.

14. Zhi D, Yang T, Yang J, Fu S, Zhang S. Targeting strategies for superparamagnetic iron oxide nanoparticles in cancer therapy. Acta Biomater. 2020;102:13-34. doi:10.1016/j.actbio.2019.11.027

15. Yan H, Xue Z, Xie J, et al. Toxicity of carbon nanotubes as anti-tumor drug carriers. Int J Nanomedicine. 2019;14:10179-10194.

16. Akbarzadeh A, Rezaei-Sadabady R, Davaran S, et al. Liposome: classification, preparation, and applications. Nanoscale Res Lett. 2013;8(1):102.

17. Sercombe L, Veerati T, Moheimani F, Wu SY, Sood AK, Hua S. Advances and challenges of liposome assisted drug delivery. Front Pharmacol. 2015;6:286.

18. Loureiro JA, Gomes B, Coelho MA, Do Carmo Pereira M, Rocha S. Immunoliposomes doubly targeted to transferrin receptor and to alpha-synuclein. Future Sci OA. 2015;1(4):FSO71. doi:10.4155/fso.15.71

19. Danhier F, Ansorena E, Silva JM, Coco R, Le Breton A, Préat V. PLGAbased nanoparticles: an overview of biomedical applications. J Controlled Release. 2012;161(2):505-522. doi:10.1016/j.jconrel.2012.01.043

20. Song X, Wang J, Xu Y, Shao H, Gu J. Surface-modified PLGA nanoparticles with PEG/LA-chitosan for targeted delivery of arsenic trioxide for liver cancer treatment: inhibition effects enhanced and side effects reduced. Colloids Surf $B$ Biointerfaces. 2019;180:110-117. doi:10.1016/j.colsurfb.2019.04.036

21. Loureiro JA, Gomes B, Fricker G, Coelho MAN, Rocha S, Pereira MC. Cellular uptake of PLGA nanoparticles targeted with anti-amyloid and anti-transferrin receptor antibodies for Alzheimer's disease treatment. Colloids Surf B Biointerfaces. 2016;145:8-13.

22. Deepika MS, Thangam R, Sheena TS, et al. Dual drug loaded PLGA nanospheres for synergistic efficacy in breast cancer therapy. Mater Sci Eng C Mater Biol Appl. 2019;103:109716. doi:10.1016/j. msec.2019.05.001

23. Geszke-Moritz M, Moritz M. Solid lipid nanoparticles as attractive drug vehicles: composition, properties and therapeutic strategies. Mater Sci Eng C Mater Biol Appl. 2016;68:982-994. doi:10.1016/j. msec.2016.05.119

24. Naseri N, Valizadeh H, Zakeri-Milani P. Solid lipid nanoparticles and nanostructured lipid carriers: structure, preparation and application. Adv Pharm Bull. 2015;5(3):305-313. doi:10.15171/apb.2015.043
25. Loureiro JA, Andrade S, Duarte A, et al. Resveratrol and grape extract-loaded solid lipid nanoparticles for the treatment of Alzheimer's disease. Molecules. 2017;22:2. doi:10.3390/molecules 22020277

26. Zheng G, Zheng M, Yang B, Fu H, Li Y. Improving breast cancer therapy using doxorubicin loaded solid lipid nanoparticles: synthesis of a novel arginine-glycine-aspartic tripeptide conjugated, $\mathrm{pH}$ sensitive lipid and evaluation of the nanomedicine in vitro and in vivo. Biomedicine \& Pharmacotherapy = Biomedecine \& Pharmacotherapie. 2019; 116:109006. doi:10.1016/j.biopha.2019.109006

27. Guney Eskiler G, Cecener G, Dikmen G, Egeli U, Tunca B. Solid lipid nanoparticles: reversal of tamoxifen resistance in breast cancer. Eur J Pharm Sci. 2018;120:73-88. doi:10.1016/j.ejps.2018.04.040

28. Mishra B, Patel BB, Tiwari S. Colloidal nanocarriers: a review on formulation technology, types and applications toward targeted drug delivery. Nanomedicine. 2010;6(1):9-24. doi:10.1016/j.nano.2009. 04.008

29. Helgason T, Awad TS, Kristbergsson K, McClements DJ, Weiss J. Effect of surfactant surface coverage on formation of solid lipid nanoparticles (SLN). J Colloid Interface Sci. 2009;334(1):75-81. doi:10.1016/j.jcis.2009.03.012

30. Loureiro JA, Gomes B, Fricker G, et al. Dual ligand immunoliposomes for drug delivery to the brain. Colloids Surf B Biointerfaces. 2015;134:213-219. doi:10.1016/j.colsurfb.2015.06.067

31. Ramalho MJ, Loureiro JA, Coelho MAN, Pereira MC. Factorial design as a tool for the optimization of PLGA nanoparticles for the co-delivery of temozolomide and O6-benzylguanine. Pharmaceutics. 2019;11:8. doi:10.3390/pharmaceutics11080401

32. Stetefeld J, McKenna SA, Patel TR. Dynamic light scattering: a practical guide and applications in biomedical sciences. Biophys Rev. 2016;8(4):409-427.

33. Keenan CM, Baker JF, Bradley AE, et al. International Harmonization of Nomenclature and Diagnostic Criteria (INHAND) progress to date and future plans. J Toxicol Pathol. 2015;28 (1):51-53. doi:10.1293/tox.2014-0049

34. Wohlfart S, Gelperina S, Kreuter J. Transport of drugs across the blood-brain barrier by nanoparticles. J Control Release. 2012;161 (2):264-273. doi:10.1016/j.jconrel.2011.08.017

35. Hoshyar N, Gray S, Han H, Bao G. The effect of nanoparticle size on in vivo pharmacokinetics and cellular interaction. Nanomedicine (Lond). 2016;11(6):673-692. doi:10.2217/nnm.16.5

36. Gaumet M, Vargas A, Gurny R, Delie F. Nanoparticles for drug delivery: the need for precision in reporting particle size parameters. Eur J Pharm Biopharm. 2008;69(1):1-9. doi:10.1016/j. ejpb.2007.08.001

37. Blanco E, Shen H, Ferrari M. Principles of nanoparticle design for overcoming biological barriers to drug delivery. Nat Biotechnol. 2015;33(9):941-951. doi:10.1038/nbt.3330

38. Danaei M, Dehghankhold M, Ataei S, et al. Impact of particle size and polydispersity index on the clinical applications of lipidic nanocarrier systems. Pharmaceutics. 2018;10:2. doi:10.3390/pharmaceutics1002 0057

39. Ong SG, Chitneni M, Lee KS, Ming LC, Yuen KH. Evaluation of extrusion technique for nanosizing liposomes. Pharmaceutics. 2016;8:4.

40. Bhattacharjee S. DLS and zeta potential - What they are and what they are not? J Control Release. 2016;235:337-351. doi:10.1016/j. jconrel.2016.06.017

41. Gelperina S, Maksimenko O, Khalansky A, et al. Drug delivery to the brain using surfactant-coated poly(lactide-co-glycolide) nanoparticles: influence of the formulation parameters. Eur J Pharm Biopharm. 2010;74(2):157-163. doi:10.1016/j.ejpb.2009.09.003

42. Scholer N, Olbrich C, Tabatt K, Muller RH, Hahn H, Liesenfeld O. Surfactant, but not the size of solid lipid nanoparticles (SLN) influences viability and cytokine production of macrophages. Int J Pharm. 2001;221(1-2):57-67. doi:10.1016/S0378-5173(01)00660-3 
43. Morris A. Microbiota drives sex-specific differences. Nat Rev Endocrinol. 2018;15(1):4.

44. Mauvais-Jarvis F, Arnold AP, Reue KA. Guide for the design of pre-clinical studies on sex differences in metabolism. Cell Metab. 2017;25(6):1216-1230. doi:10.1016/j.cmet.2017.04.033

45. Scotland RS, Stables MJ, Madalli S, Watson P, Gilroy DW. Sex differences in resident immune cell phenotype underlie more efficient acute inflammatory responses in female mice. Blood. 2011;118 (22):5918-5927. doi:10.1182/blood-2011-03-340281

46. Uner M, Yener G. Importance of solid lipid nanoparticles (SLN) in various administration routes and future perspectives. Int J Nanomedicine. 2007;2(3):289-300.

47. Sonavane G, Tomoda K, Makino K. Biodistribution of colloidal gold nanoparticles after intravenous administration: effect of particle size Colloids Surf B Biointerfaces. 2008;66(2):274-280. doi:10.1016/j. colsurfb.2008.07.004

48. Kim JH, Kim JH, Kim KW, Kim MH, Yu YS. Intravenously administered gold nanoparticles pass through the blood-retinal barrier depending on the particle size, and induce no retinal toxicity. Nanotechnology. 2009;20(50):505101. doi:10.1088/0957-4484/20/ 50/505101

49. Chen YS, Hung YC, Liau I, Huang GS. Assessment of the in vivo toxicity of gold nanoparticles. Nanoscale Res Lett. 2009;4 (8):858-864. doi:10.1007/s11671-009-9334-6

50. Cho WS, Kim S, Han BS, Son WC, Jeong J. Comparison of gene expression profiles in mice liver following intravenous injection of 4 and $100 \mathrm{~nm}$-sized PEG-coated gold nanoparticles. Toxicol Lett. 2009;191(1):96-102.

51. De Jong WH, Hagens WI, Krystek P, Burger MC, Sips AJ, Geertsma RE. Particle size-dependent organ distribution of gold nanoparticles after intravenous administration. Biomaterials. 2008;29(12):1912-1919. doi:10.1016/j.biomaterials.2007.12.037

52. Semmler-Behnke M, Kreyling WG, Lipka J, et al. Biodistribution of 1.4- and 18-nm gold particles in rats. Small. 2008;4(12):2108-2111.

53. Zhang XD, Wu HY, Wu D, et al. Toxicologic effects of gold nanoparticles in vivo by different administration routes. Int J Nanomedicine. 2010;5:771-781. doi:10.2147/IJN.S8428

54. Koziara JM, Lockman PR, Allen DD, Mumper RJ. In situ blood-brain barrier transport of nanoparticles. Pharm Res. 2003;20 (11):1772-1778. doi:10.1023/B:PHAM.0000003374.58641.62
55. Lockman PR, Mumper RJ, Khan MA, Allen DD. Nanoparticle technology for drug delivery across the blood-brain barrier. Drug Dev Ind Pharm. 2002;28(1):1-13.

56. Vergoni AV, Tosi G, Tacchi R, Vandelli MA, Bertolini A, Costantino L. Nanoparticles as drug delivery agents specific for CNS: in vivo biodistribution. Nanomedicine. 2009;5(4):369-377. doi:10.1016/j.nano.2009.02.005

57. Cho WS, Cho M, Jeong J, et al. Acute toxicity and pharmacokinetics of 13 nm-sized PEG-coated gold nanoparticles. Toxicol Appl Pharmacol. 2009;236(1):16-24.

58. Balasubramanian SK, Jittiwat J, Manikandan J, Ong CN, Yu LE, Ong WY. Biodistribution of gold nanoparticles and gene expression changes in the liver and spleen after intravenous administration in rats. Biomaterials. 2010;31(8):2034-2042. doi:10.1016/j.biomaterials. 2009.11.079

59. Balogh L, Nigavekar SS, Nair BM, et al. Significant effect of size on the in vivo biodistribution of gold composite nanodevices in mouse tumor models. Nanomedicine. 2007;3(4):281-296. doi:10.1016/j. nano.2007.09.001

60. Oberdorster G, Maynard A, Donaldson K, et al. Principles for characterizing the potential human health effects from exposure to nanomaterials: elements of a screening strategy. Part Fibre Toxicol. 2005;2:8. doi:10.1186/1743-8977-2-8

61. Choi J, Rui Y, Kim J, et al. Nonviral polymeric nanoparticles for gene therapy in pediatric CNS malignancies. Nanomedicine. 2020;23:102115. doi:10.1016/j.nano.2019.102115

62. Pinheiro RGR, Granja A, Loureiro JA, et al. Quercetin lipid nanoparticles functionalized with transferrin for Alzheimer's disease. Eur J Pharm Sci. 2020;148:105314. doi:10.1016/j.ejps.2020.105314

63. Gupta R, Xie H. Nanoparticles in daily life: applications, toxicity and regulations. J Environ Pathol Toxicol Oncol. 2018;37(3):209-230. doi:10.1615/JEnvironPatholToxicolOncol.2018026009

64. Jeevanandam J, Barhoum A, Chan YS, Dufresne A, Danquah MK. Review on nanoparticles and nanostructured materials: history, sources, toxicity and regulations. Beilstein $J$ Nanotechnol. 2018;9:1050-1074.
International Journal of Nanomedicine

\section{Publish your work in this journal}

The International Journal of Nanomedicine is an international, peerreviewed journal focusing on the application of nanotechnology in diagnostics, therapeutics, and drug delivery systems throughout the biomedical field. This journal is indexed on PubMed Central, MedLine, CAS, SciSearch ${ }^{\mathbb{R}}$, Current Contents ${ }^{\mathbb{B}} /$ Clinical Medicine,
Journal Citation Reports/Science Edition, EMBase, Scopus and the Elsevier Bibliographic databases. The manuscript management system is completely online and includes a very quick and fair peer-review system, which is all easy to use. Visit http://www.dovepress.com/ testimonials.php to read real quotes from published authors. 